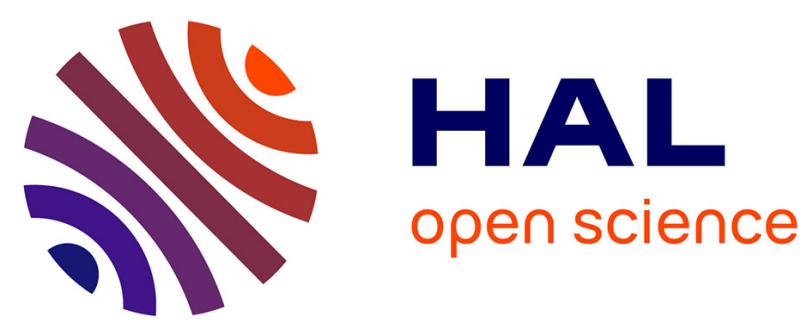

\title{
Visualization of subsurface damage in woven carbon fiber-reinforced composites using polarization-sensitive terahertz imaging
}

Junliang Dong, Pascal Pomarède, Lynda Chehami, Alexandre Locquet, Fodil Meraghni, Nico F. Declercq, D.S. Citrin

\section{To cite this version:}

Junliang Dong, Pascal Pomarède, Lynda Chehami, Alexandre Locquet, Fodil Meraghni, et al.. Visualization of subsurface damage in woven carbon fiber-reinforced composites using polarization-sensitive terahertz imaging. NDT \& E International, 2018, 99, pp.72-79. 10.1016/j.ndteint.2018.07.001 . hal02317518

\section{HAL Id: hal-02317518 \\ https://hal.science/hal-02317518}

Submitted on 16 Oct 2019

HAL is a multi-disciplinary open access archive for the deposit and dissemination of scientific research documents, whether they are published or not. The documents may come from teaching and research institutions in France or abroad, or from public or private research centers.
L'archive ouverte pluridisciplinaire HAL, est destinée au dépôt et à la diffusion de documents scientifiques de niveau recherche, publiés ou non, émanant des établissements d'enseignement et de recherche français ou étrangers, des laboratoires publics ou privés. 


\title{
Visualization of subsurface damage in woven carbon fiber-reinforced composites using polarization-sensitive terahertz imaging
}

\author{
Junliang Dong ${ }^{\mathrm{a}, \mathrm{b}, *}$, Pascal Pomarède ${ }^{\mathrm{c}, \mathrm{b}}$, Lynda Chehami ${ }^{\mathrm{b}}$, Alexandre Locquet ${ }^{\mathrm{b}, \mathrm{a}}$, Fodil Meraghni ${ }^{\mathrm{c}}$, \\ Nico F. Declercq ${ }^{\mathrm{d}, \mathrm{b}}$, D.S. Citrin ${ }^{\mathrm{a}, \mathrm{b}, * *}$ \\ ${ }^{\text {a }}$ School of Electrical and Computer Engineering, Georgia Institute of Technology, Atlanta, GA 30332-0250, USA \\ ${ }^{\mathrm{b}}$ Georgia Tech-CNRS UMI2958, Georgia Tech Lorraine, 2 Rue Marconi, 57070 Metz, France \\ ' Arts et metiers ParisTech, LEM3 UMR CNRS 7239, 4 Rue Augusstin Fresnel, 57078 Metz, France \\ ${ }^{\mathrm{d}}$ Woodruff School of Mechanical Engineering, Georgia Institute of Technology, Atlanta, GA 30332, USA
}

\section{A R T I C L E I N F O}

\section{Keywords:}

Terahertz imaging

Carbon fiber-reinforced composites

Subsurface damage

Terahertz polarization

Deconvolution

\begin{abstract}
A B S T R A C T
Polarization-sensitive terahertz imaging is applied to characterize subsurface damage in woven carbon fiberreinforced composite laminates in this study. Terahertz subsurface spectral imaging based on terahertz deconvolution is tailored and applied to detect, in a nondestructive fashion, the subsurface damage within the first ply of the laminate caused by a four-point bending test. Subsurface damage types, including matrix cracking, fiber distortion/fracture, as well as intra-ply delamination, are successfully characterized. Our results show that, although the conductivity of carbon fibers rapidly attenuates terahertz propagation with depth, the imaging capability of terahertz radiation on woven carbon fiber-reinforced composites can nonetheless be significantly enhanced by taking advantage of the terahertz polarization and terahertz deconvolution. The method demonstrated in this study is capable of extracting and visualizing a number of fine details of the subsurface damage in woven carbon fiber-reinforced composites, and the results achieved are confirmed by comparative studies with X-ray tomography.
\end{abstract}

\section{Introduction}

Fiber-reinforced composites, which combine the merits of high strength and low weight, offer an alternative to conventional structural materials, such as metals, and are widely used for various applications including aerospace, automotive, renewable energy and marine industry. As a result, the wide applicability of fiber-reinforced composites has created the correspondingly need for advanced nondestructive evaluation (NDE) techniques for inspection and failure detection during manufacturing and maintenance. Currently, for many materials and applications, there is no adequate NDE technique available to detect important damage types that occur at depth but may not be visually evident at the surface. Given the potential catastrophic nature of failure in some of the aforementioned applications, there has been an intense search for NDE techniques that might provide the desired information.

Terahertz (THz) imaging, which can provide a non-invasive, noncontact, and non-ionizing modality to characterize numerous nonmetallic materials, is emerging as a relatively new promising NDE technique for fiber-reinforced composites. The $\mathrm{THz}$ portion of the electromagnetic spectrum, extends from approximately $100 \mathrm{GHz}$ to $10 \mathrm{THz}$, and lies between the microwave and infrared; the corresponding wavelength range is from $30 \mu \mathrm{m}$ to $3 \mathrm{~mm}$. THz waves can penetrate various nonmetallic materials that may be opaque in the range of visible and infrared light. Moreover, as nonionizing radiation, $\mathrm{THz}$ waves present no known health risks.

$\mathrm{THz}$ imaging was first applied to the NDE of fiber-reinforced composites in 2006 [1] and has been explored extensively since [2]. For composites reinforced by glass fibers, $\mathrm{THz}$ waves can be utilized to characterize both surface and underlying damage and defects, including mechanical/heat damage [3], voids [4], delamination [5,6], intrusions and moisture contamination [7]. Material parameters [8] and fiber orientation [9] in glass fiber-reinforced composites have also been successfully characterized via $\mathrm{THz}$ imaging. Composites reinforced by other non-conductive fibers, such as ultra-high molecular weight polyethylene (UHMWPE) composites, have also been explored with THz imaging for characterization of projectile impact damage [10,11]. However, the studies of $\mathrm{THz}$ imaging of carbon fiber-reinforced composites are quite limited, mainly due to the conductivity of carbon 
fibers, which hinders the penetration of THz radiation at depth. Thus far, to our knowledge, THz imaging has mainly been applied to detect the damage on the surface of unidirectional carbon fiber-reinforced composites [12,13]. Although carbon fibers hinder the THz penetration, the interaction between the $\mathrm{THz}$ polarization and carbon fiber orientation can be utilized to extract detailed information which cannot be obtained by other NDE techniques. In our previous work [14], polarization-resolved $\mathrm{THz}$ imaging has been successfully applied to a hybrid fiber-reinforced composite laminate containing glass-fibers/ epoxy and carbon-fibers/epoxy plies with a cross-ply stack pattern. Impact-induced inter-ply damage at the interface and intra-ply damage close to the same interface can be differentiated by taking advantage of the sensitivity of $\mathrm{THz}$ polarization to the anisotropic conductivity of unidirectional carbon fibers.

In this study, for the first time to our knowledge, we demonstrate how to characterize subsurface damage in woven carbon fiber-reinforced composite laminates, which is of great potential importance to the aerospace and automotive industries, via polarization-sensitive $\mathrm{THz}$ imaging. THz subsurface spectral imaging based on $\mathrm{THz}$ deconvolution is developed and utilized to extract the detailed subsurface damage of several types within the first ply of the laminate caused by a four-point bending test. Subsurface damage types, including matrix cracking, fiber distortion/fracture, and intra-ply delamination, can be clearly distinguished. A comparative study with X-ray tomography is also performed to confirm the results obtained from $\mathrm{THz}$ imaging.

\section{Theoretical background and method}

\subsection{THz polarization and carbon-fiber orientation}

The electrical conductivity of carbon fibers severely limits the penetration of $\mathrm{THz}$ waves into composites. Generally, carbon fiber-reinforced composite laminates can be classified into unidirectional laminates and woven (multidirectional) laminates. Considering one ply of unidirectional carbon fiber-reinforced composite laminate, the electrical conductivity $\sigma$ is anisotropic and depends on the THz polarization and fiber orientation, which can be expressed as [15].

$\sigma(\theta)=\sigma_{l} \cos ^{2} \theta+\sigma_{t} \sin ^{2} \theta$,

where $\theta$ is the angle between the THz polarization and fiber orientation, and $\sigma_{l}$ and $\sigma_{t}$ are the longitudinal and transverse conductivities, respectively. Along the fiber direction, the electric current flows through the carbon fibers, so the longitudinal conductivity $\sigma_{l}$ depends on the conductivity of carbon fibers $\sigma_{f}$ and on the fiber volume fraction $\nu_{f}$,

$\sigma_{l} \approx \sigma_{f} \nu_{f}$.

For the transverse conductivity $\sigma_{t}$, because the resin material is nonconductive, the flow of electric current only occurs due to random contact between adjacent carbon fibers, which depends on the manufacturing process and the quality of the composites [15]. Therefore, the longitudinal conductivity is much higher than the transverse conductivity. Then we can further conclude that (1) when the THz polarization is parallel to the orientation of carbon fibers, the electric conductivity is maximum and the $\mathrm{THz}$ reflectivity also reaches its maximum; (2) when the THz polarization is perpendicular to the carbon fibers, conductivity and reflectivity achieve their minimum. In principle, THz reflective imaging can be utilized as an effective method to characterize impact damage by monitoring the reflection coefficient across the surface of unidirectional carbon fiber-reinforced composites. Suffering from impact, carbon fiber distortion and fracture will occur in the damaged area, which will lead to spatial variations of the reflection coefficient as well as the polarization anisotropy. The reflection coefficient in regions with and without impact damage can be more easily distinguished with polarization parallel to the carbon-fiber orientation [13].

For woven carbon fiber-reinforced composite laminates, the analysis of the electrical conductivity is more complicated. Besides the electrical properties of the constituent materials and the fiber volume fraction, the electrical conductivity of woven carbon fiber-reinforced composites also depends on the weave pattern, and the contacts between the adjacent tows with different fiber directions in the ply $[16,17]$. Therefore, the interaction between the $\mathrm{THz}$ polarization and woven carbon-fiber structure should be investigated before performing $\mathrm{THz}$ imaging. Proper selection of the $\mathrm{THz}$ polarization is able to produce enhanced contrasts for the $\mathrm{THz}$ imaging results, which we will show in the following.

\section{2. $\mathrm{THz}$ subsurface spectral imaging}

Our focus in this study is on THz subsurface spectral imaging, which is designed to extract subsurface damage in woven carbon fiber-reinforced composite laminates. Although $\mathrm{THz}$ waves are strongly attenuated while propagating through one ply of woven carbon fiber-reinforced composite, the waves do provide significant subsurface information, especially when the composite suffers damage, THz waves may penetrate deeper and produce echoes when damage is encountered. Therefore, although not superficially evident, the raw reflected THz signals are the superposition of the echo reflected from the surface and temporally overlapping subtle reflections from subsurface features. In order to highlight the subsurface information, it is necessary to separate subsurface echoes and the surface echo using deconvolution techniques.

Our previous work has demonstrated the power of THz deconvolution to resolve overlapping echoes associated with closely-spaced features in depth [18-20]. In short, THz deconvolution aims to reconstruct the impulse-response function, that is intrinsic to the sample and independent of the $\mathrm{THz}$ pulse employed, which should ideally consist of a sequence of ideal impulses corresponding to reflections from the interfaces of a stratified system [21]. With THz deconvolution, the depth-resolution of $\mathrm{THz}$ imaging can be greatly enhanced. By exploiting the sparse constraint, i.e., the expectation that only a limited number of temporal data points have nonzero values in the impulseresponse function, THz sparse deconvolution developed in Ref. [22], is able to provide a clear representation of the impulse-response function with sharp pulses, and increase the depth-resolution to $\sim 45 \mu \mathrm{m}$ in air given the spectral bandwidth of our apparatus.

Exploiting sparse deconvolution, $\mathrm{THz}$ subsurface spectral imaging can be performed by using the following procedure: (1) retrieve the deconvolved signals, corresponding to the impulse-response function, by applying $\mathrm{THz}$ sparse deconvolution to the received raw signals; in the deconvolved signals, the peaks indicating the location of surface and subsurface echoes are well-resolved; (2) identify the initial peak corresponding to the surface echo, and subtract this peak from the deconvolved signals, then the remaining deconvolved signals only contain the subsurface information; (3) re-convolve the remaining deconvolved signals with the $\mathrm{THz}$ reference signal to obtain the $\mathrm{THz}$ subsurface time-domain echoes; (4) perform the Fourier transform of the THz subsurface echoes, and integrate the frequency components in the higher frequency range of the available spectral bandwidth to form the imaging contrast; because higher frequency components, corresponding to shorter wavelength, are thus retained, the resulting images enhance both the depth and transverse resolution.

\section{Sample and experiment}

Two samples were investigated in this study. Both are nominally identical polyphenylene-sulfide-based composite laminates, reinforced with eight plies of woven carbon fibers. The dimensions of the laminates are $160 \mathrm{~mm}$ (warp direction, $x$ ) $\times 25 \mathrm{~mm}$ (weft direction, y) $\times 2.4 \mathrm{~mm}$ (thickness direction, $z$ ), shown in Fig. 1 (a). The weave pattern of carbon fibers is $2 / 1$ twill with warp-dominated surface, and the structure of the samples is layered as the sequence 


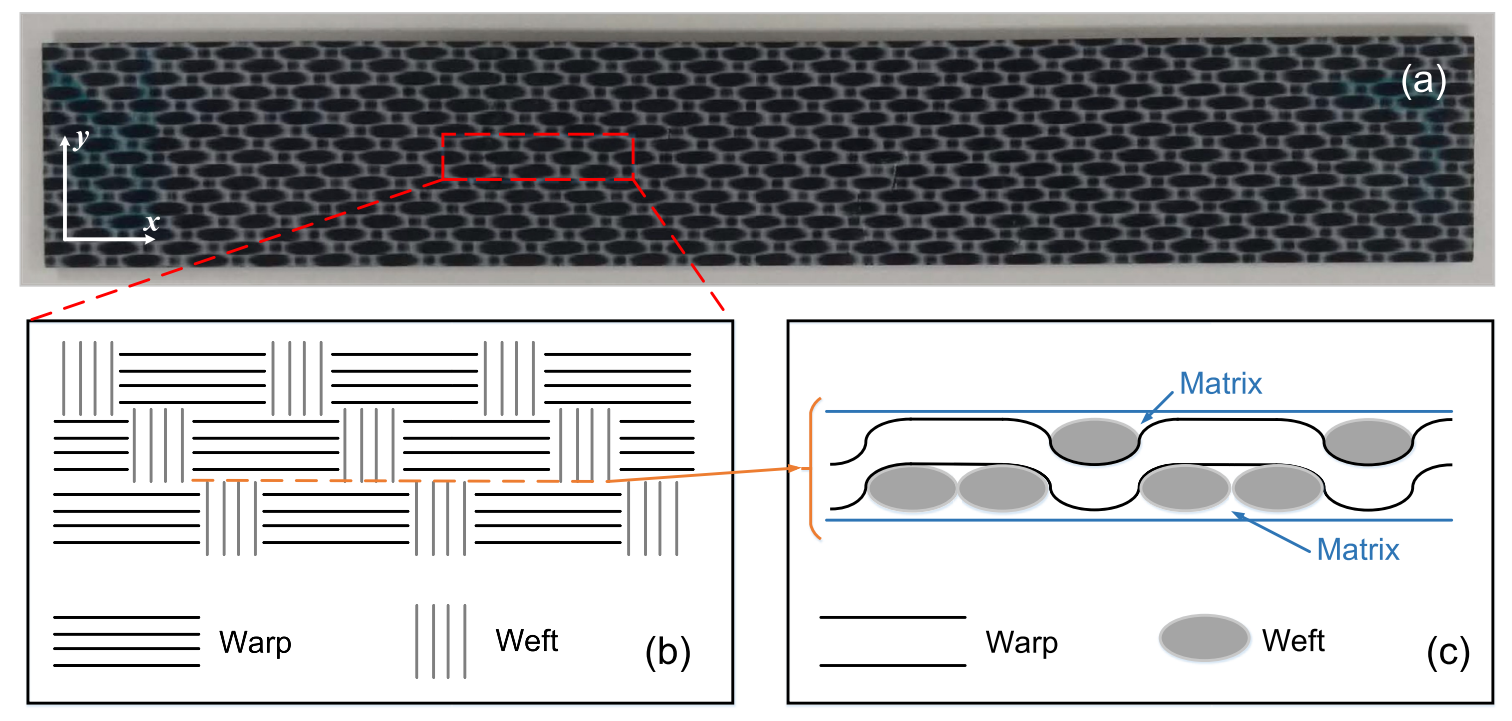

Fig. 1. The typical sample investigated in this study. (a) Optical photograph of the sample; (b) in-plane schematic of the 2/1 twill weave pattern; (c) cross-sectional schematic of the sample ply.

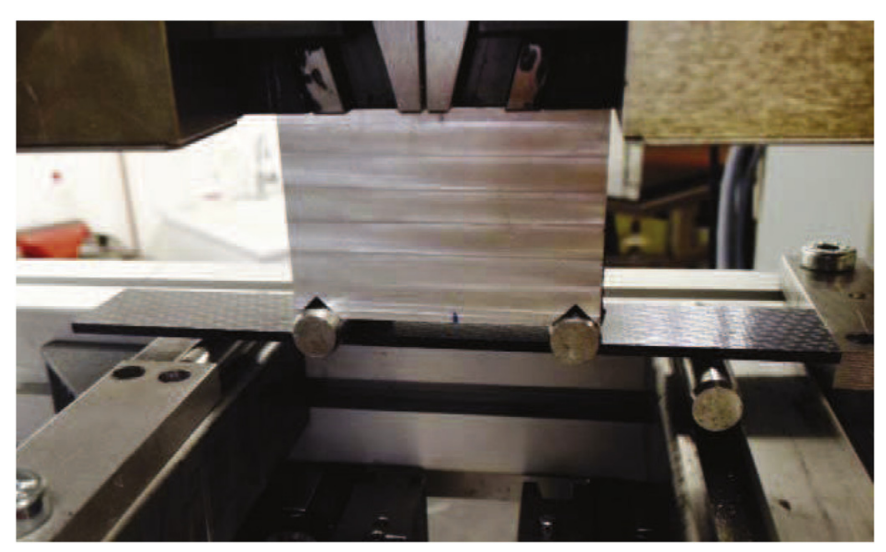

Fig. 2. Photograph of the four-point bending test. Note the carbon fiber-reinforced composite sample placed horizontally near the center of the image. The dimensions of the laminates are $160 \mathrm{~mm}$ (warp direction) $\times 25 \mathrm{~mm}$ (weft direction) $\times 2.4 \mathrm{~mm}$ (thickness direction).

$\left[0^{\circ} / 90^{\circ},-45^{\circ} / 45^{\circ}\right]_{2 s}$. The schematic structure of the first ply of this woven carbon fiber-reinforced composites is illustrated in Fig. 1(b) and (c). The volume fraction of carbon fiber in the composite laminate is about $50 \%$ and the Young's modulus of the samples is about $56 \mathrm{GPa}$ along the fiber's axes in both warp and weft directions. The damage was introduced by four-point bending tests, shown in Fig. 2, with two different conditions. With the four-point bending test, we are able to introduce various damage types by tension and compression, which are useful for investigating different damage mechanisms. Sample 1 was subjected to the four-point test under one-cycle static loading with the applied force of $1200 \mathrm{~N}$; Sample 2 was subjected to four-point bending test under 500 cycles static loading with the applied force of $800 \mathrm{~N}$. The distance between the rollers on the top surface (compression surface) was about $50 \mathrm{~mm}$, and the distance between the rollers on the bottom surface (tension surface) was about $105 \mathrm{~mm}$.

The commercial $\mathrm{THz}$ time-domain spectroscopy (TDS) system (Teraview TPS Spectra 3000), which was employed in this study, is shown schematically in Fig. 3(a). The GaAs photoconductive antenna is excited by an ultrafast (femtosecond) laser to produce roughly singlecycle THz pulses with bandwidth extending from $60 \mathrm{GHz}$ to $3 \mathrm{THz}$. The ultrafast laser used here is an Er-doped fiber laser that emits $780 \mathrm{~nm}$ pulses with sub-100 fs pulse duration at a repetition rate of $100 \mathrm{MHz}$ and has an average output power in excess of $65 \mathrm{~mW}$. Coherent detection of the $\mathrm{THz}$ radiation is performed in a similar photoconductive antenna circuit. By gating the photoconductive gap with a femtosecond pulse synchronized to the $\mathrm{THz}$ emission, a current proportional to the $\mathrm{THz}$ electric field is measured. By varying the optical path length, the THz time-domain waveform can be sampled, resulting in both amplitude and phase information on a sub-picosecond timescale.

$\mathrm{THz}$ reflective imaging was performed at an angle of incidence of $\sim 10^{\circ}$. Before scanning the samples, the $\mathrm{THz}$ reference signal was recorded by setting a metal plate at the sample position. The time-domain waveform and the frequency spectrum of this reference signal are shown in Fig. 3(b). The THz pulse in the reference signal is composed of roughly one electromagnetic cycle, and its duration is a few picoseconds. The spectrum of the reference signal, obtained by Fourier transforming the temporal pulse, extends from $\sim 100 \mathrm{GHz}$ to $\sim 3 \mathrm{THz}$. The ringing after the main $\mathrm{THz}$ pulse are due to ambient water-vapor and the pronounced atmospheric water-absorption lines are clearly observed in the spectrum. The focus spot size of the THz beam is frequency-dependent, and is about $300 \mu \mathrm{m}$ at $1 \mathrm{THz}$. The samples were raster-scanned by a set of motorized stages moving in $x$ (warp) and $y$ (weft) directions with a $0.2 \mathrm{~mm}$ spatial step size over the center area of the top surface (subject to compression due to the four-point bending test) of the sample. Each recorded temporal $\mathrm{THz}$ waveform contains 2048 data points, and the data sampling period was set to $0.02325 \mathrm{ps}$. The signal was averaged over 10 shots per pixel to enhance the signalto-noise ratio. The raster-scan was conducted in a temperature-controlled laboratory at $22^{\circ} \mathrm{C}$, and the humidity in the laboratory was held about $38 \%$. In order to consider the interaction between the THz polarization and carbon-fiber orientation, the $\mathrm{THz}$ polarization was set first along the warp direction; after completing the scan, the polarization was rotated and set along the weft direction. Therefore, for each sample, two sets of 3D volumetric raw data were acquired.

\section{Results and discussion}

First, we present the $\mathrm{THz}$ spectral images based on the raw $\mathrm{THz}$ signals directly obtained from the raster-scanning. Note that the raw $\mathrm{THz}$ signals are dominated by the initial reflection from the sample surface, and thus the images so obtained will provide mainly nearsurface information. Later, we consider images obtained by windowingout the initial surface reflection to reveal more clearly subsurface information.

The contrast mechanism $M$ of THz C-scans in the frequency domain 

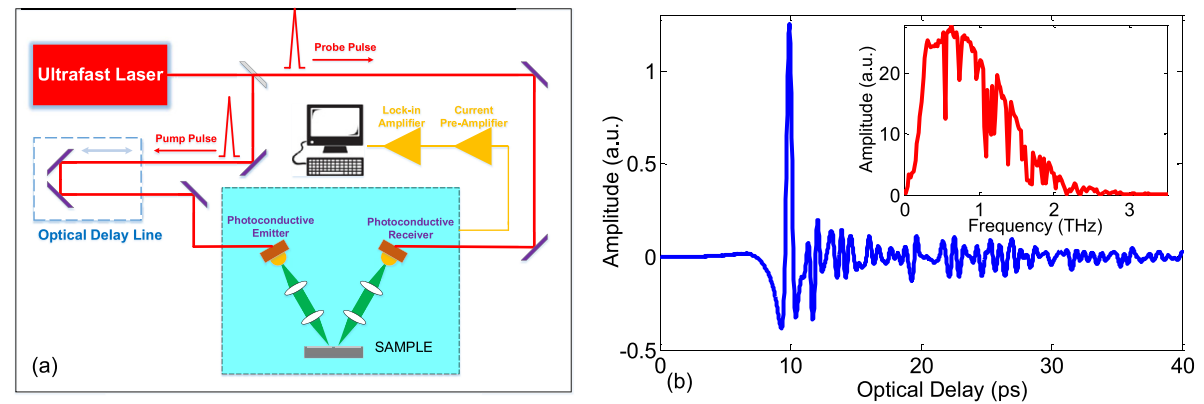

Fig. 3. THz time-domain spectroscopy (TDS) system. (a) Schematic of the THz TDS system in reflection configuration. (b) THz reference signal with its spectrum in the inset.

is obtained by taking the Fourier transforms of the raw reflected $\mathrm{THz}$ waveform $E(t)$ at each pixel, and integrating its magnitude in a specified frequency window, which can be expressed as,

$M=\int_{f_{1}}^{f_{2}}|\mathcal{F}(E(t))| d f$,

where $\mathcal{F}$ represents the Fourier transform, $f_{1}$ and $f_{2}$ are the limits of the frequency window. In order to reveal the small and subtle features, the cutoffs are restricted to relatively high frequencies $f_{1}=1.0 \mathrm{THz}$ to $f_{2}=3.0 \mathrm{THz}$ in this study. The contrasts in THz C-scans in this study are all normalized to one (maximum). THz C-scans plotted based on this contrast mechanism should present both the surface and subsurface information since we here include the initial reflected peak from the air-sample interface.

THz C-scans based on raw THz signals for Sample 1 with THz polarization along the warp and weft directions are shown in Fig. 4 (a) and (b), respectively. With $\mathrm{THz}$ polarization along the warp direction, the THz C-scan in Fig. 4(a) clearly shows the warp-tow pattern on the sample surface, since warp-tows provide the highest $\mathrm{THz}$ reflection and therefore, dominate the contrast. The weft-tow pattern cannot be observed, which indicates the $\mathrm{THz}$ reflection from weft-tows is much smaller than that from warp-tows. Besides the warp-tow pattern, two regions of interest (ROI) are identified in Fig. 4(a). In ROI 1, the warptows show relatively smaller $\mathrm{THz}$ reflections than those outside ROI 1 , which indicates less $\mathrm{THz}$ reflection, and thus, more $\mathrm{THz}$ penetration in these warp-tows. This is because the compression applied, in the fourpoint bending, leads to the growth of micro-scale cracking of tows and matrix in these warp-tows [23], which lowers the electrical conductivity and thus decreases the reflectivity there. ROI 2 highlights the most severe damaged area displaying low contrast, in which the pattern of warp-tows is obscured due to the macro-scale damages, including matrix cracking, fiber distortion and fracture.

One the other hand, with THz polarization along the weft direction, in the THz C-scan in Fig. 4(b), the weave pattern, including both the warp- and weft-tows, is observed. Typical $\mathrm{THz}$ reflected signals from the warp- and weft-tows with different $\mathrm{THz}$ polarization are plotted in Fig. 5 for comparison. It is seen that, with $\mathrm{THz}$ polarization along the weft direction (in blue and red), the difference of $\mathrm{THz}$ reflections from the warp- and weft-tows is much smaller than that with THz polarization along the warp direction (in green and orange). It is also noticed that, with $\mathrm{THz}$ polarization along the weft direction, the amplitude of the THz reflected signal from the warp-tow is even a little higher than that from the weft-tow. This observation can be attributed to the fact that, due to the $2 / 1$ twill weave pattern of the sample, the area of contact between the warp-tow on the surface and the weft-tow underneath is about two times as large as the area of contact between the weft-tow and the warp-tow underneath in the ply (shown in Fig. 1(c)). A larger contact area leads to higher electrical conductivity, and therefore, larger THz reflectivity. One striking point in Fig. 4(b) is that there is a much larger area, in which the warp- and weft-tows provide relatively smaller reflections, can be identified. Again, this feature indicates the growth of micro-scale cracking within the tows. We conclude that, for our $2 / 1$ twill samples, THz polarization along the weft
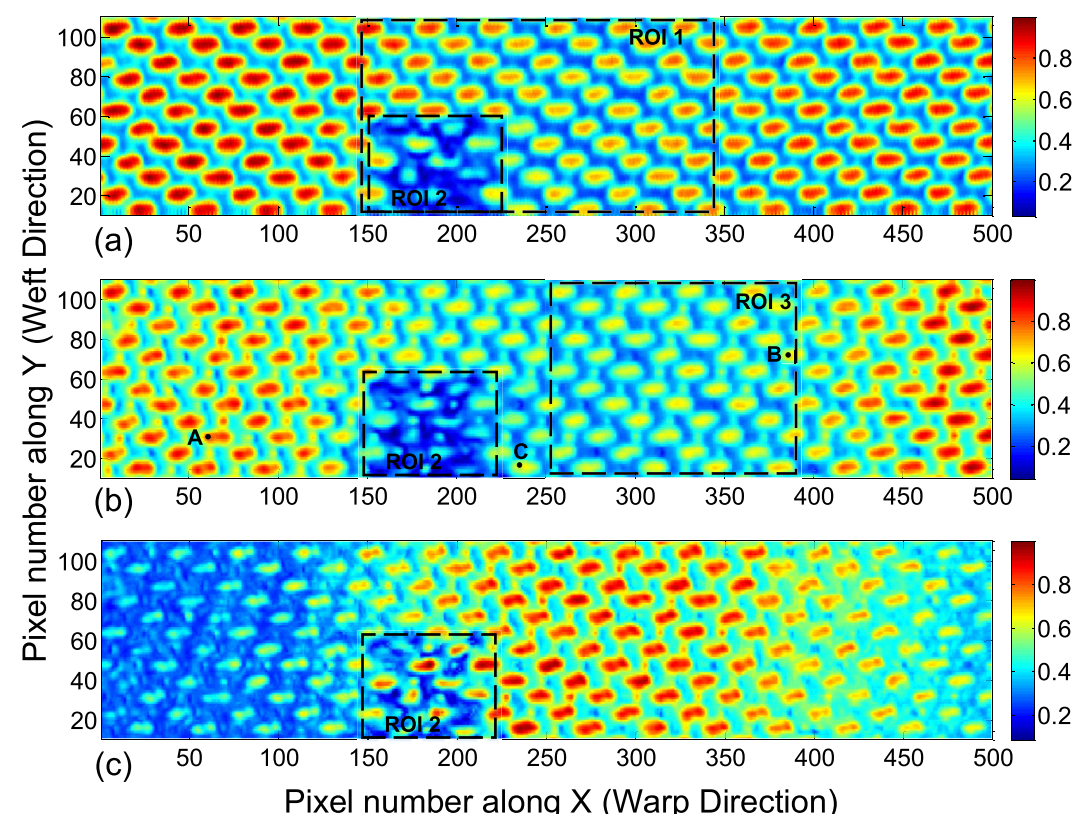

Fig. 4. THz C-scan images of Sample 1. (a) The THz C-scan based on the raw $\mathrm{THz}$ signals with $\mathrm{THz}$ polarization parallel to the warp direction. (b) The $\mathrm{THz} \mathrm{C}$-scan based on the raw $\mathrm{THz}$ signals with $\mathrm{THz}$ polarization parallel to the weft direction. (c) The THz C-scan based on the THz subsurface signals with $\mathrm{THz}$ polarization parallel to the weft direction. 


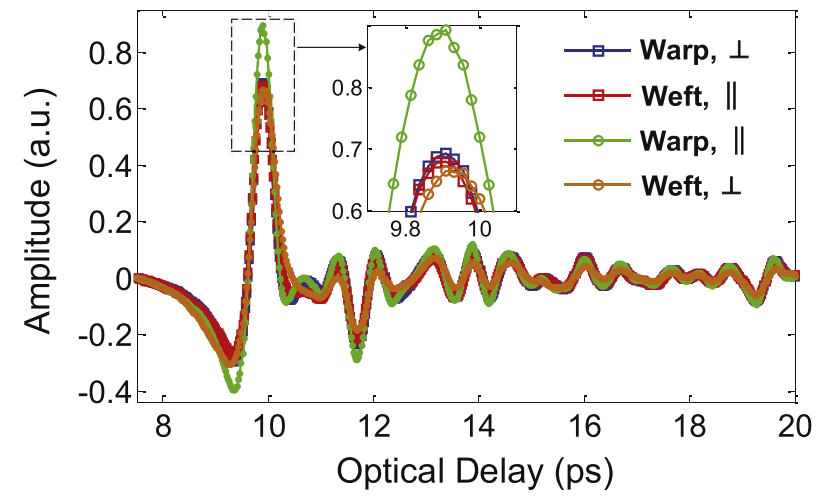

Fig. 5. Typical THz reflected signals from the warp- and weft tows with different THz polarization. Blue: THz reflection from warp-tows with polarization in the weft direction; red: $\mathrm{THz}$ reflection from weft tows with polarization in the weft direction; green: THz reflection from warp-tows with polarization in the warp direction; orange: $\mathrm{THz}$ reflection from weft-tows with polarization in the warp direction.

direction provides better imaging contrast, and is more effective in detecting the micro-scale damages within the tows. Macro-scale damages, including matrix cracking, fiber distortion and fracture, can also be detected in Fig. 4(b).

THz C-scans based on raw THz signals for Sample 2 with polarization along the warp and weft directions are shown in Fig. 6 (a) and (b), respectively. As was the case for Sample 1, with THz polarization along the weft direction, the weave pattern, including both the warp- and weft-tows, is observed. Macro-scale damage, including matrix cracking, fiber distortion and fracture can also be characterized with better contrast in ROI 4 of Fig. 6(b). ROI 5 clearly exhibits a typical damage morphology [24] within samples with twill weave under bending, in which the smaller THz reflection from the warp-tow indicates the tow failure, and the lower contrast around the failed tow indicates the growth of cracking. We emphasize that, polarization-sensitive $\mathrm{THz}$ imaging is effective in detecting various damages on the surface of woven carbon fiber-reinforced composites. The existence of micro-scale damages will reduce the strength of the $\mathrm{THz}$ reflections from the tows, and macro-scale damages will change or obscure the weave pattern.

As mentioned at the beginning of Section 4, THz C-scans based on the raw THz signals contain both surface and subsurface information; however, since the initial $\mathrm{THz}$ reflection from the surface dominates the reflected signals, the subsurface features will tend to be hidden by the surface features. In order to highlight the subsurface information, $\mathrm{THz}$ subsurface spectral imaging based on THz sparse deconvolution is utilized in the following. The process by which the subsurface images are obtained involves temporally windowing out the initial surface reflection to emphasize later features associated with regions below the surface in the reflected signal. With $\mathrm{THz}$ polarization along the weft direction, typical reflected raw $\mathrm{THz}$ signals from Sample 1, corresponding to the signals at points A $(64,30), \mathrm{B}(383,72)$, and C $(235,15)$ in Fig. 4(b), are shown in Fig. 7(a). From Point A to C, the amplitude of the first peak of raw $\mathrm{THz}$ signals, corresponding to the $\mathrm{THz}$ reflection from the surface of the laminate, decreases, which indicates successively increasing micro-scale damage progressing from Point A to B to C. More importantly, the increase of amplitude of the signals after the first peak is observed, which indicates the existence of echoes from subsurface features. The signals boxed in Fig. 7(a) are the superposition of the echoes from the subsurface features and ringing due to ambient water-vapor.

In order to extract the echoes from subsurface features, THz sparse deconvolution based on the shrinkage algorithm was performed. After deconvolution, a sparsity-based impulse-response function is obtained, which entirely depends on material structures. In the deconvolution process, we consider the $\mathrm{THz}$ reference signal as the input and the raw $\mathrm{THz}$ reflected signal as the output; therefore, the actual impulse-response function associated with reflection coefficients should be obtained by multiplying the deconvolved signal by a factor of -1 for phase correction. The impulse-response function obtained, or the deconvolved signal, consists of a baseline at zero and then a sequence of sharp peaks, which correspond to the interfaces producing the echoes. The details of the algorithm can be found in Ref. [22].

The deconvolved signals achieved by sparse deconvolution are shown in Fig. 7(b). The first positive peaks with the maximum amplitude, corresponding to the echoes from the surface, are clearly identified. From Point A to C, the amplitude of the first positive peaks decreases, which also indicates the increase of the micro-scale damage. There are small negative peaks located before the main peaks, which are attributed to the diffuse reflections (the back scattering of waves from a rough surface, on which the waves are scattered at many angles rather than at just one angle in the case of specular reflection) due to

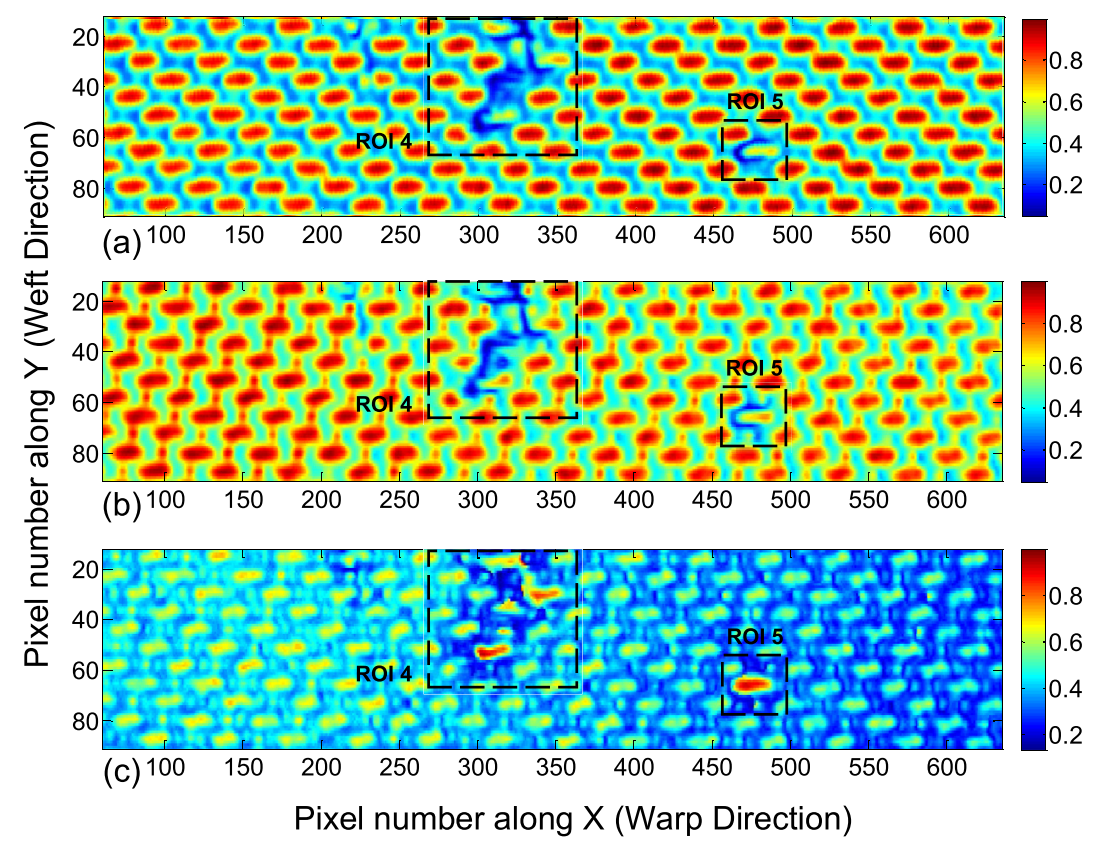

Fig. 6. THz C-scan images of Sample 2. (a) THz C-scan in the frequency domain based on the raw $\mathrm{THz}$ signals with $\mathrm{THz}$ polarization parallel to the warp direction. (b) THz C-scan in the frequency domain based on the raw $\mathrm{THz}$ signals with $\mathrm{THz}$ polarization parallel to the weft direction. (c) THz C-scan in the frequency domain based on the THz subsurface signals with THz polarization parallel to the weft direction. 

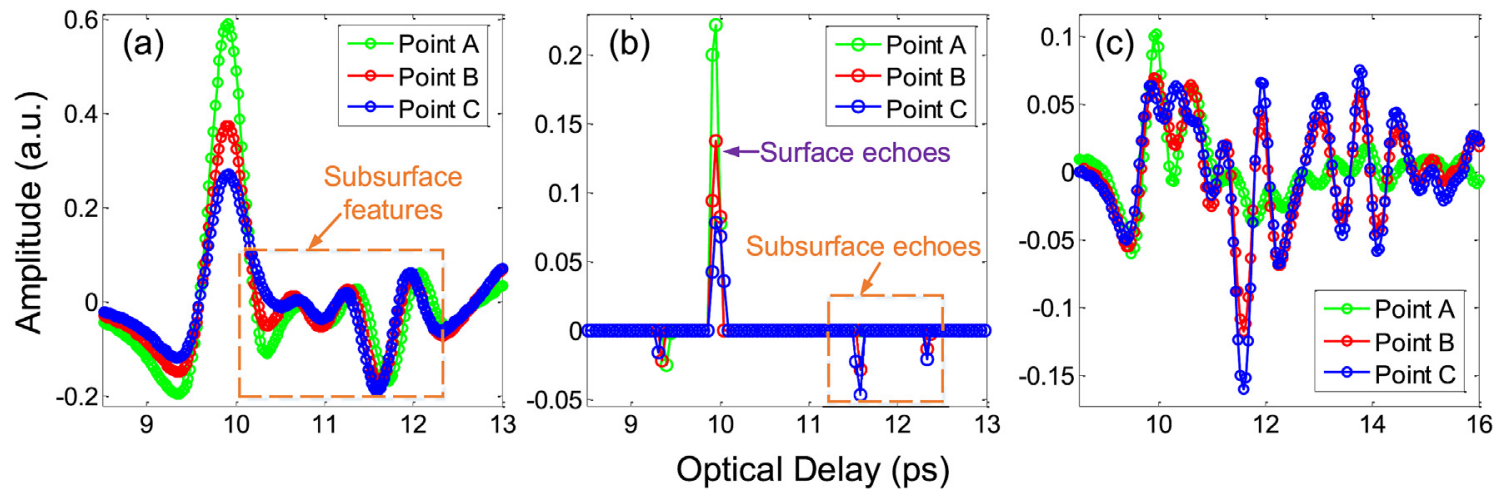

Fig. 7. Typical THz raw, deconvolved, and subsurface signals from Sample 1 with THz polarization along the weft direction. (a) THz reflected raw signals. (b) THz deconvolved signals based on sparse deconvolution. (c) THz subsurface echoes achieved by re-convolution following temporally windowing out the initial surface echo in the reflected signals. Notice that the horizontal scale in (c) differs that in (a) and (b).
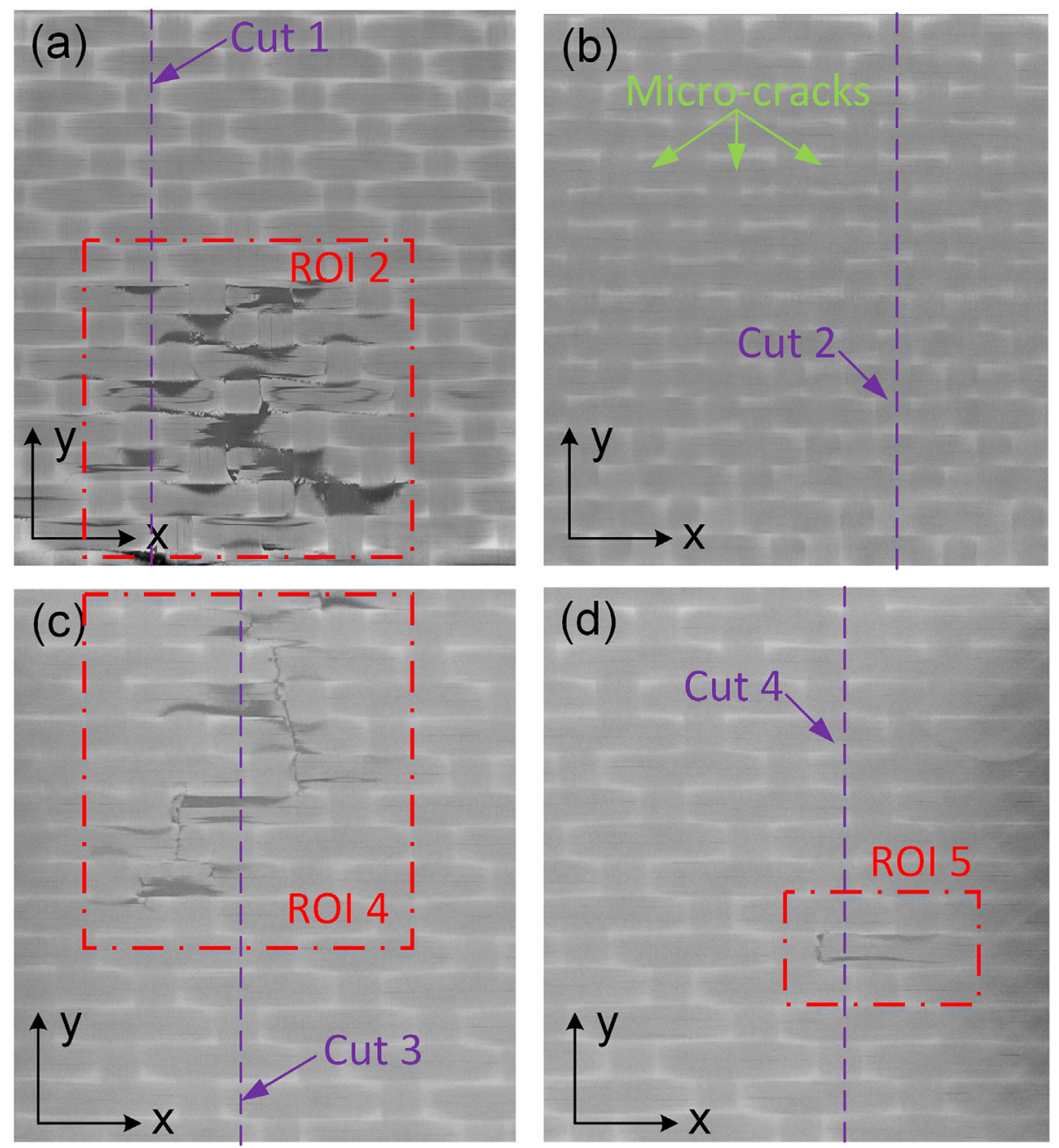

Fig. 8. Surface images of Sample 1 and 2 obtained by X-ray tomography, corresponding to (a) ROI 2 in Fig. 4, (b) ROI 3 in Fig. 4, (c) ROI 4 in Fig. 6 , and (d) ROI 5 in Fig. 6, respectively.

the inherent uneven surface of the woven-fiber fabric. The most important features are, at Points B and C, small negative peaks that are successfully resolved after the first positive peaks, confirming the existence of $\mathrm{THz}$ echoes originating from features in the subsurface region. In order to eliminate the $\mathrm{THz}$ reflection from the surface and recover the time-domain subsurface echoes, the first positive peaks in the deconvolved signals are temporally windowed out, and the time-domain subsurface echoes are obtained by the convolution of the remaining deconvolved signals with the $\mathrm{THz}$ reference signal. The subsurface echoes recovered are shown in Fig. 7(c). It is observed that the amplitude of the subsurface echoes at Point $C$ is larger than that Point B. There are two physical origins of the subsurface echoes: (1) due to the growth of micro-cracking in tows, more $\mathrm{THz}$ power penetrates into the ply, and $\mathrm{THz}$ echoes are produced when $\mathrm{THz}$ waves encounter the interface between the warp- and weft-tows within the ply; (2) the growth of delamination induced by the four-point bending test will 
produce THz echoes with larger amplitude, due to the existence of an air gap. Therefore, the amplitude of the subsurface echoes can be used to evaluate the severity of damage, as higher amplitude indicates the occurrence of delamination. For example, we can conclude that there is delamination at Point C, since the amplitude of subsurface echoes are significantly larger there.

Based on all the subsurface echoes extracted from the 3D volume data, THz subsurface spectral imaging was performed based on Eq. (3), and the subsurface images for Sample 1 and 2 are shown in Figs. 4(c) and Fig.6(c), respectively. In Fig. 4(c), we observe that areas with high contrast mainly occur under the warp-tows, because the growth of micro-cracking mainly occurs in the warp-tows, and more $\mathrm{THz}$ power penetrates there that subsequently bounces back at the interface between warp- and weft-tows. Areas with highest contrast, especially in and around ROI 2, indicate the existence of delamination, corresponding to intra-ply delamination [24]. In Fig. 6(c), areas with delamination are also highlighted with highest contrast. The delamination seen in ROI 5 confirms the damage evolution, as the cracking propagates around the failed tow will inevitably seed the formation of delamination. Another striking point in Fig. 6(c) is that, compared with the damage pattern in Fig. 6(b), larger regions with lower contrast in ROI 4, corresponding to the marco-scale damages in matrix and tows, can be identified. Based on the subsurface images, we conclude that $\mathrm{THz}$ subsurface spectral imaging is indeed effective in revealing the fine details of subsurface damage.

Comparison with X-ray micro-computed tomography was carried out to confirm the results from $\mathrm{THz}$ imaging. An EasyTom system manufactured by $R X$ solutions was used. The voxel size of the acquisitions was set to $12 \mu \mathrm{m}$ for both samples. After the images acquisitions, a 3D reconstruction was done with the $X$-Act software from $R X$ solutions for each sample. The obtained volumes were finally investigated using the image processing software Avizo. Surface images of typical areas in Sample 1 and 2 obtained by X-ray tomography are depicted in Fig. 8, corresponding to the various ROIs in the THz images. Fig. 8(a) and (c) clearly exhibit the macro-scale damages, including the matrix cracking, fiber distortion and fracture in ROI 2 and ROI 4. Besides the macroscale damages, X-ray tomography is also able to identify the micro-scale damages. In Fig. 8(b), corresponding to ROI 3 in Fig. 4, the growth of micro-cracking, shown as dim horizontal lines, can be observed in almost every warp-tow. Due to the way the bending was applied in this study, micro-cracking mainly occurs in warp-tows, and propagates along warps-tows. The growth of micro-cracking, considered as the first stage of damage evolution, can also be observed in warp-tows in Fig. 8(a), (c), and (d). Fig. 8(d) shows the same damage morphology in ROI 5 as what is described in Fig. 6. Cross-sectional images obtained by
X-ray tomography are shown in Fig. 9, corresponding to typical cutslices highlighted in Fig. 8. Fig. 9(a), (c), and (d) mainly show the existence of delaminations in the corresponding ROIs; the formation of delamination mainly occurs at the interface between the warp-tow and weft-tow underneath within the first ply, corresponding to the intra-ply delamination. The growth of transverse micro-cracking is confirmed and identified again in Fig. 9(b). In conclusion, the images obtained by $\mathrm{X}$-ray tomography supports and confirms our analysis of $\mathrm{THz}$ images.

\section{Conclusion}

In this study, $\mathrm{THz}$ reflective imaging is utilized to characterize damage in woven carbon fiber-reinforced composite laminates subject to four-point bending tests. We first demonstrate how to take the advantage of $\mathrm{THz}$ polarization and $\mathrm{THz}$ deconvolution technique to enhance the capability of $\mathrm{THz}$ imaging on woven carbon fiber-reinforced composites given the high $\mathrm{THz}$ attenuation in these materials, and to extract the fine details of the subsurface damages.

We show that, besides the interaction between $\mathrm{THz}$ polarization and carbon-fiber orientation, the weave pattern and the contacts between the tows will also influence the THz reflection across the surface, and further influence the imaging contrasts in $\mathrm{THz} \mathrm{C}$-scans. For the samples with $2 / 1$ twill weave pattern in this study, THz polarization along the weft direction provides better contrast, and is more effective in detecting the micro-scale damage compared with $\mathrm{THz}$ polarization along the warp direction.

An analysis technique is developed to enhance the ability of $\mathrm{THz}$ NDE to reveal subsurface damage in these materials, whereas many previous $\mathrm{THz}$ studies have yielded little useful subsurface information due to the low penetration of $\mathrm{THz}$ waves within these materials. $\mathrm{THz}$ subsurface spectral imaging based on THz sparse deconvolution is utilized to extract the subsurface echoes and highlight the subsurface damages which cannot be identified by the conventional $\mathrm{THz}$ imaging. Subsurface damage types, including the matrix cracking, fiber distortion and fracture, as well as intra-ply delamination have been successfully identified. Due to its sensitivity to carbon fibers, THz imaging is able to clearly show the weave pattern of carbon fibers, and the occurrence of both micro-scale and macro-scale damages can be identified. Our results prove that the enhanced THz imaging can be utilized to characterize both the surface and subsurface damages in woven carbon fiber-reinforced composites, and should be considered as a complimentary NDE modality to investigate the damage mechanisms of woven carbon fiber-reinforced composites.

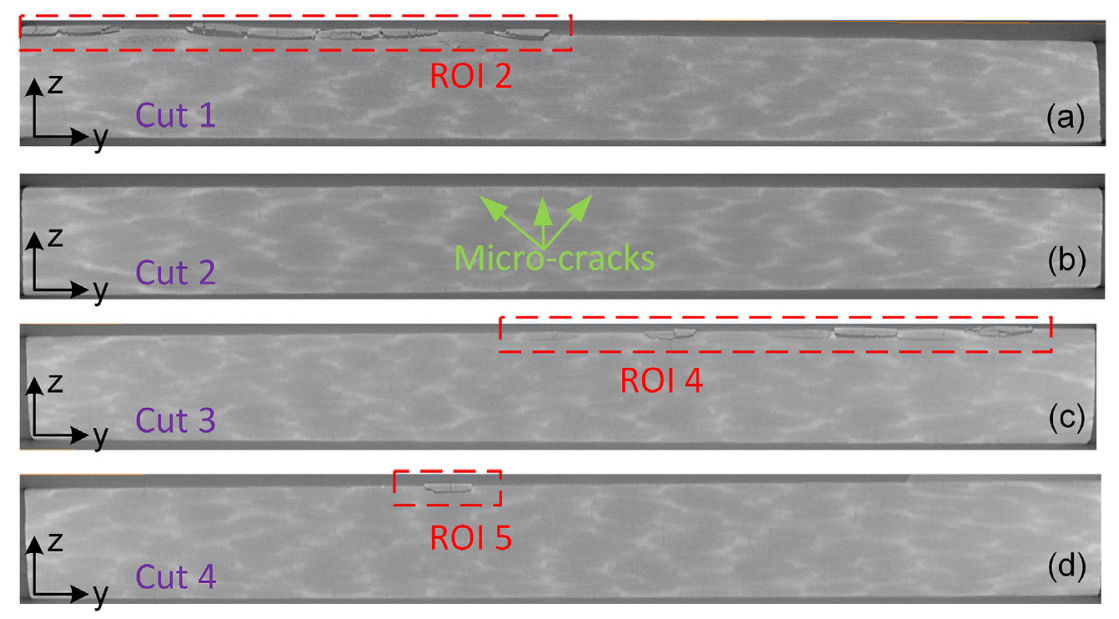

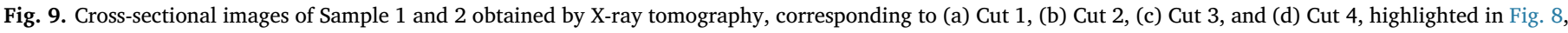
respectively. 


\section{Acknowledgment}

The authors gratefully acknowledge the financial support of the Conseil Régional du Grand Est of the Fonds Européen de Développement Régional (FEDER), and of the Institut Carnot ARTS.

\section{Appendix A. Supplementary data}

Supplementary data related to this article can be found at http://dx. doi.org/10.1016/j.ndteint.2018.07.001.

\section{References}

[1] Rutz F, Koch M, Khare S, Moneke M, Richter H, Ewert U. Terahertz quality control of polymeric products. Int J Infrared Millimet Waves 2006;27:547-56.

[2] Amenabar I, Lopez F, Mendikute A. In introductory review to THz non-destructive testing of composite mater. J Infrared, Millim Terahertz Waves 2013;34:152169.

[3] Stoik CD, Bohn MJ, Blackshire JL. Nondestructive evaluation of aircraft composites using transmissive terahertz time domain spectroscopy. Optic Express 2008;16:17039.

[4] Stoik CD, Bohn MJ, Blackshire JL. Nondestructive evaluation of aircraft composites using reflective terahertz time domain spectroscopy. NDT Int 2010;43:106115.

[5] Dong J, Kim B, Locquet A, McKeon P, Declercq N, Citrin DS. Nondestructive evaluation of forced delamination in glass fiber-reinforced composites by terahertz and ultrasonic waves. Compos B Eng 2015;79:667675.

[6] Dong J, Locquet A, Citrin DS. Enhanced terahertz imaging of small forced delamination in woven glass fibre-reinforced composites with wavelet de-noising. J Infrared, Millim Terahertz Waves 2016;37:289301.

[7] Ospald F, Zouaghi W, Beigang R, Matheis C, Jonuscheit J, Recur B, Guillet J-P, Mounaix P, Vleugels W, Bosom PV, Gonzlez LV, Lpez I, Edo RM, Sternberg Y, Vandewal M. Aeronautics composite material inspection with a terahertz time-domain spectroscopy system. Opt Eng 2013;53:31208.

[8] Naito K, Kagawa Y, Utsuno S, Naganuma T, Kurihara K. Dielectric properties of woven fabric glass fiber reinforced polymer-matrix composites in the THz frequency range. Compos Sci Technol 2009;69:20272029.

[9] Jördens C, Scheller M, Wietzke S, Romeike D, Jansen C, Zentgraf T, et al. Terahertz spectroscopy to study the orientation of glass fibres in reinforced plastics. Compos Sci Technol 2010;70(3):472-7.

[10] Palka N, Panowicz R, Ospald F, Beigang R. 3D non-destructive imaging of punctures in polyethylene composite armor by THz time domain spectroscopy. J Infrared, Millim Terahertz Waves 2015;36:770788.

[11] Palka N, Panowicz R, Chalimoniuk M, Beigang R. Non-destructive evaluation of puncture region in polyethylene composite by terahertz and X-ray radiation. Compos B Eng 2016;92:315325.

[12] Nesterov PK, Yachin VV, Zinenko TL, Kuleshov YM. Characterization of CFRP thermal degradation by the polarization-frequency reflectometry method in subterahertz frequency range. IEEE Trans. Terahertz Sci. Technol 2016;6:9198.

[13] Yang S-H, Kim K-B, Oh HG, Kang J-S. Non-contact detection of impact damage in CFRP composites using millimeter-wave reflection and considering carbon fiber direction. NDT E Int 2013;57(0):45-51.

[14] Dong J, Locquet A, Declercq NF, Citrin DS. Polarization-resolved terahertz imaging of intra- and inter-laminar damages in hybrid fiber-reinforced composite laminate subject to low-velocity impact. Compos B Eng 2016;92:167174.

[15] Athanasopoulos N, Kostopoulos V. Prediction and experimental validation of the electrical conductivity of dry carbon fiber unidirectional layers. Compos B Eng 2011;42:15781587.

[16] Athanasopoulos N, Kostopoulos V. A comprehensive study on the equivalent electrical conductivity tensor validity for thin multidirectional carbon fibre reinforced plastics. Compos B Eng 2014;67:244255.

[17] Senghor FD, Wasselynck G, Bui HK, Branchu S, Trichet D, Berthiau G. Electrical conductivity tensor modeling of stratified woven-fabric carbon fiber reinforced polymer composite materials. IEEE Trans Magn 2017;53:14.

[18] Dong J, Bianca Jackson J, Melis M, Giovanacci D, Walker GC, Locquet A, Bowen JW, Citrin DS. Terahertz frequency-wavelet domain deconvolution for stratigraphic and subsurface investigation of art painting. Optic Express 2016;24:26972.

[19] Dong J, Locquet A, Melis M, Citrin DS. Global mapping of stratigraphy of an oldmaster painting using sparsity-based terahertz reflectometry. Sci Rep 2017;7:15098.

[20] Dong J, Locquet A, Citrin DS. Terahertz quantitative nondestructive evaluation of failure modes in polymer-coated steel. IEEE J Sel Top Quant Electron 2017;23:17.

[21] Dong J, Locquet A, Citrin DS. Depth resolution enhancement of terahertz deconvolution by autoregressive spectral extrapolation. Opt Lett 2017;42:1828.

[22] Dong J, Wu X, Locquet A, Citrin DS. Terahertz superresolution stratigraphic char acterization of multilayered structures using sparse deconvolution. IEEE Trans. Terahertz Sci. Technol 2017;7:260267.

[23] Mortell DJ, Tanner DA, McCarthy CT. An experimental investigation into multiscale damage progression in laminated composites in bending. Compos Struct 2016;149:3340

[24] De Carvalho NV, Pinho ST, Robinson P. An experimental study of failure initiation and propagation in 2D woven composites under compression. Compos Sci Technol 2011;71:13161325. 